\title{
Application of a hybrid least squares algorithm in astronomical positioning
}

\author{
ZHANG Xihui, ZHOU Zhaofa, LIU Xianyi, ZHU Wenyong
}

Rocket Force University of Engineering, Xi'an Shanxi, 710025,China.

785564025@qq.com

Keywords: Hybrid, squares algorithm, Astronomical positioning

\begin{abstract}
In order to reduce the digital zenith camera identify stars data that exist in the gross error influence on astronomical calculating, improve positioning accuracy. The mapping relation between the CCD image coordinate system and the celestial tangent plane coordinate system is established by using the robust weighted hybrid least squares algorithm. Using the least squares algorithm did not consider of the errors in the coefficient matrix and the data of the gross error. So the least squares algorithm and the total least squares algorithm are effectively combined to form the hybrid least squares algorithm. Then the hybrid least squares algorithm is used for robust weighting, reduced the identify stars possible gross error in the data, and reasonable weight matrix is set in conjunction with the magnitude. Experiments show that the robust weighted hybrid least squares algorithm has high accuracy in astronomical solution.
\end{abstract}

\section{INTRODUCTION}

When using digital zenith camera positioning used Helmet model for conversion between coordinates $^{[1]}$. The coordinates of the CCD image of the star and the coordinates of the celestial plane have some errors, and in the coordinate transformation parameters to solve the time often use the least squares algorithm ${ }^{[2]}$. The least squares algorithm only considers the error of the observation, but does not take into account the error of the coefficient matrix ${ }^{[3]}$, the overall least squares algorithm can take into account the error of the coefficient matrix and the observation ${ }^{[4]}$,but the values in the overall least squares coefficient matrix contain some errors. In fact, there is a constant series (or row) with no errors in the coefficient matrix.In order to identify the coordinates of the star's coordinate transformation parameters with high precision,the least squares algorithm and the total least squares algorithm are combined to form a mixed least squares algorithm. The mixed least squares algorithm takes into account both the constant series (or row) in the matrix and the errors in the coefficient matrix and observations ${ }^{[5][6]}$. However, there may be a rough error in the data, Considering the poor ability of the mixed least squares algorithm to resist the coarse error ${ }^{[7]}$, a robust weighted mixed least squares algorithm is proposed, the weighted star data is weighted and the influence of the coarse error on the solution parameters is weakened.

\section{COORDINATE TRANSFORMATION MODEL}

In the process of positioning with a digital zenith camera meter, the star map of the zenith is photographed by the CCD star sensor. Through the star table for star map recognition, the establishment of the stars to determine the celestial plane coordinate system and CCD image coordinate system, the conversion between the two coordinates using Helmet conversion model, there are:

$$
\left[\begin{array}{l}
u \\
v
\end{array}\right]=\left[\begin{array}{cc}
-a & b \\
b & a
\end{array}\right]\left[\begin{array}{l}
x \\
y
\end{array}\right]+\left[\begin{array}{l}
c_{1} \\
c_{2}
\end{array}\right]
$$

Where $(u, v)$ is the tangent plane coordinate that identifies the star on the celestial sphere, the tangent plane coordinates are obtained by recognizing the red plate of the star. $(x, y)$ is the coordinates of the CCD image identifying the star, by reading the CCD image coordinates. $a 、 b 、$ 
$C_{1} 、 C_{2}$ is the coordinate conversion parameter.Make:

$$
\begin{aligned}
& \boldsymbol{I}=\left[\begin{array}{llllll}
u_{1} & \cdots & u_{n} & v_{1} & \cdots & v_{n}
\end{array}\right]^{T} \\
& \boldsymbol{A}=\left[\begin{array}{cccc}
1 & 0 & -x_{1} & y_{1} \\
\vdots & \vdots & \vdots & \vdots \\
1 & 0 & -x_{n} & y_{n} \\
0 & 1 & y_{1} & x_{1} \\
\vdots & \vdots & \vdots & \vdots \\
0 & 1 & y_{n} & x_{n}
\end{array}\right]
\end{aligned}
$$

When the number of identified star points is large, the equation (1) can be expressed as

$$
\boldsymbol{I}=\boldsymbol{A x}
$$

Where $\boldsymbol{A}$ is a matrix of coefficients consisting of CCD image coordinates that identify stars, and $\boldsymbol{I}$ is a matrix of tangent planes. $\boldsymbol{X}$ is the desired parameter $\left[c_{1}, c_{2}, a, b\right]^{T}$. In order to be able to accurately calculate the model parameters, here using the least squares algorithm.

\section{SOLUTION OF COORDINATE TRANSFORMATION PARAMETERS IN ASTRONOMICAL POSITIONING}

\section{Least Squares Algorithm}

When the number of identified star points is greater than the number of solving parameters, the least squares algorithm is used to solve the parameters. The least squares algorithm takes into account the error of the observation. The residual value is expressed by $\boldsymbol{V}$, there are

$$
\boldsymbol{V}=\boldsymbol{A} \boldsymbol{x}-\boldsymbol{I}
$$

$\boldsymbol{P}$ is the vector of the observed vector, using the least squares algorithm

$$
\boldsymbol{V}^{T} \boldsymbol{P V}=\min
$$

The value of the solvable parameter $\hat{x}$

$$
\hat{\boldsymbol{x}}=\left(\boldsymbol{A}^{T} \boldsymbol{P A}\right)^{-1} \boldsymbol{A}^{T} \boldsymbol{P} \boldsymbol{I}
$$

The prerequisite for solving the parameters using the least squares method is that there is no error in the coefficient matrix $\boldsymbol{A}$, but the coefficient matrix $\boldsymbol{A}$ is made up of the CCD image coordinates of the identification star in the process of positioning with the digital zenith meter. In the process of reading the image coordinates will produce a certain amount of error, that is, using the least squares algorithm can not eliminate the CCD image star point caused by the error, so the direct use of the least squares algorithm is unreasonable.

\section{Total Least Squares Algorithm}

The overall least squares are an algorithm that can take into account both the coefficient matrix and the errors in the observations. In the process of parameter solving, the total least squares algorithm should be used when the coefficient matrix and the observation quantity contain certain errors.

For equations

$$
A x=l
$$

When both the coefficient matrix and the observations contain errors, the equations can be expressed as:observations contain errors, the equations can be expressed as:

$$
\left(\boldsymbol{A}+\boldsymbol{E}_{A}\right) \boldsymbol{x}=\boldsymbol{I}+\boldsymbol{E}_{\boldsymbol{I}}
$$

The overall least squares algorithm solves the value of parameter $x$ in $\min \|[\boldsymbol{A}, \boldsymbol{I}]-[\hat{\boldsymbol{A}}, \hat{\boldsymbol{I}}]\|_{F}$ condition. In the process of positioning with the digital zenith, the coordinates of the celestial plane and the coordinates of the CCD image are $\left(u_{i}, v_{i}\right)$ and $\left(x_{i}, y_{i}\right)$ respectively. The weight matrix of the celestial plane and the coordinates of the CCD image are $\boldsymbol{P}_{1}$ and $\boldsymbol{P}_{2}$, respectively. That is, the 
covariance matrix is $\boldsymbol{Q}_{1}=\boldsymbol{P}_{1}^{-1}$ and $\boldsymbol{Q}_{2}=\boldsymbol{P}_{2}^{-1}$ respectively. The amount of error can be expressed as

$$
\boldsymbol{V}_{4 n \times 1}=\left[\begin{array}{c}
\boldsymbol{v}_{1} \\
\boldsymbol{v}_{2}
\end{array}\right]=\left[v_{u 1}, v_{v 1}, \cdots, v_{x 1}, v_{y 1}, \cdots\right]^{T}
$$

Where $v_{1}$ is the error corresponding to the identification of the star in the tangent plane of the celestial sphere, and $v_{2}$ is the corresponding error in identifying the coordinates of the star in the CCD image. The criteria for the overall least squares estimation can be expressed as

$$
\boldsymbol{v}_{1}^{T} \boldsymbol{P}_{1} \boldsymbol{v}_{1}+\boldsymbol{v}_{2}^{T} \boldsymbol{P}_{2} \boldsymbol{v}_{2}=\min
$$

In the star map matching may be due to star center of mass error or matching errors and other reasons to identify the star data contains coarse errors, the existence of coarse errors will directly affect the final accuracy of the parameters of the solution. While the overall least squares algorithm is less resistant to coarse errors. So the direct use of the overall least squares can not effectively eliminate the effect of coarse errors on the parameters.

\section{Robust Weighted Mixed Least Squares Algorithm}

\section{Mixed least squares algorithm}

The least squares algorithm is combined with the overall least squares algorithm to form a mixed least squares algorithm, which can take into account the errors of the coefficient matrix and the observation vector, and can take into account the constant columns in the coefficient matrix.

The coefficient matrix $\boldsymbol{A}$ and the conversion parameter $\boldsymbol{x}$ are decomposed into:

$$
\begin{aligned}
& \boldsymbol{A}=\left[\boldsymbol{A}_{1}, \boldsymbol{A}_{2}\right], \boldsymbol{A}_{1} \in R^{m \times n_{1}}, \boldsymbol{A}_{2} \in R^{n_{2}} \\
& \boldsymbol{x}=\left[\boldsymbol{x}_{1}{ }^{T}, \boldsymbol{x}_{2}{ }^{T}\right]^{T}, \boldsymbol{x}_{1} \in R^{n_{1}}, \boldsymbol{x}_{2} \in R^{n_{2}}
\end{aligned}
$$

Where $m$ is the number of observations, $n$ is the number of parameters to be estimated, $\boldsymbol{A}_{1}$ is the constant column without error, and $n_{1}$ and $n_{2}$ are the number of parameters corresponding to matrices $\boldsymbol{A}_{1}$ and $\boldsymbol{A}_{2}$, respectively. The equations can be expressed as:

$$
A_{1} x_{1}+\left(A_{2}+E_{A_{2}}\right) x_{2}=l+E_{l}
$$

$\mathrm{QR}$ decomposition method is used to solve the equations, $\mathrm{QR}$ decomposition of the coefficient $A_{1}$

$$
\boldsymbol{A}_{1}=\boldsymbol{Q} \boldsymbol{R}_{1}
$$

Will be $\boldsymbol{Q}^{T}$ left by the equation group

$$
\boldsymbol{Q}^{T}\left[\boldsymbol{A}_{1}, \boldsymbol{A}_{2}\right]\left[\boldsymbol{x}_{1}^{T}, \boldsymbol{x}_{2}^{T}\right]^{T}=\boldsymbol{Q}^{T} \boldsymbol{I}
$$

There is a matrix

$$
\boldsymbol{C}=\left[\boldsymbol{Q}^{T} \boldsymbol{A}_{1}, \boldsymbol{Q}^{T} \boldsymbol{A}_{2}, \boldsymbol{Q}^{T} \boldsymbol{I}\right]=\left[\begin{array}{ccc}
\boldsymbol{R}_{11} & \boldsymbol{R}_{12} & \boldsymbol{R}_{11} \\
\mathbf{0} & \boldsymbol{R}_{22} & \boldsymbol{R}_{21}
\end{array}\right]
$$

So the original equations can be decomposed as:

$$
\begin{aligned}
& \boldsymbol{R}_{11} \boldsymbol{x}_{1}+\boldsymbol{R}_{12} \boldsymbol{x}_{2}=\boldsymbol{R}_{1 l} \\
& \boldsymbol{R}_{22} \boldsymbol{x}_{2}=R_{2 l}
\end{aligned}
$$

The total least squares algorithm is used to solve $\boldsymbol{x}_{2}$ to obtain the parameter estimate $\hat{\boldsymbol{x}_{2}}$, and $\hat{\boldsymbol{x}_{2}}$ is substituted into the above equation and the least value is used to solve the estimated value $\hat{\boldsymbol{x}}_{1}$ of the parameter $\boldsymbol{x}_{1}$. Finally available

$$
\begin{aligned}
& \hat{\boldsymbol{x}_{1}}=\boldsymbol{R}_{11}{ }^{-1}\left(\boldsymbol{R}_{1 l}-\boldsymbol{R}_{12} \boldsymbol{x}_{2 t l s}\right) \\
& \hat{\boldsymbol{x}_{2}}=\boldsymbol{x}_{2 t l s}
\end{aligned}
$$

In the formula, $x_{2 t s}$ is the result of solving the least squares algorithm.

\section{Robust Weighted Mixed Least Squares Algorithm}

When using the mixed least squares algorithm to solve the model parameters, the coarse error in 
the star data is not taken into account. And coarse error will have a greater impact on the parameters of the solution. So the hybrid least squares algorithm for robust weighting. The expression of the mixed least squares residual value is:

$$
\boldsymbol{V}=\frac{\boldsymbol{b}-\boldsymbol{A} x}{\sqrt{1+\left(\boldsymbol{x}_{2}\right)^{T} \boldsymbol{x}_{2}}}
$$

The parameters of the robust mixed least squares algorithm are:

$$
\begin{gathered}
\hat{\boldsymbol{v}^{i}}=\frac{\left(\boldsymbol{b}-\boldsymbol{A} \hat{\boldsymbol{x}}^{i}\right)^{T} \boldsymbol{P}(\boldsymbol{b}-\boldsymbol{A} \hat{\boldsymbol{x}})}{1+\left(\hat{\boldsymbol{x}}_{2}^{i}\right)^{T} \hat{\boldsymbol{x}}^{i}} \\
\hat{\boldsymbol{x}}_{1}^{i+1}=-\left(\boldsymbol{A}_{1}^{T} \boldsymbol{A}_{1}\right)^{-1}\left(\boldsymbol{A}_{1}^{T} \boldsymbol{A}_{2} \hat{\boldsymbol{x}}_{2}-\boldsymbol{A}_{1}^{T} \boldsymbol{b}\right) \\
\hat{\boldsymbol{x}}_{2}^{i+1}=\left(\boldsymbol{A}_{2}^{T} \boldsymbol{P} \boldsymbol{A}_{2}-\boldsymbol{A}_{2}^{T} \boldsymbol{P} \boldsymbol{A}_{1}\left(\boldsymbol{A}_{1}^{T} \boldsymbol{P} \boldsymbol{A}_{1}\right)^{-1} \bullet \boldsymbol{A}_{1}^{T} \boldsymbol{P} \boldsymbol{A}_{2}\right)^{-1} \bullet\left(\boldsymbol{A}_{2}^{T} \boldsymbol{P} \boldsymbol{b}-\boldsymbol{A}_{2}^{T} \boldsymbol{P} \bullet \boldsymbol{A}_{1}\left(\boldsymbol{A}_{1}^{T} \boldsymbol{P} \boldsymbol{A}_{1}\right)^{-1} \boldsymbol{A}_{1}^{T} \boldsymbol{P} \boldsymbol{b}+\hat{\boldsymbol{x}}_{2} \hat{\boldsymbol{v}}^{i}\right)
\end{gathered}
$$

Where $P$ is the weight coefficient matrix and the parameters calculated by the least squares algorithm are used as the initial values to do the above calculation until $\left\|x_{2}^{i+1}-x_{2}^{i}\right\|<\varepsilon$ 。

\section{DATA ANALYSIS}

During the calculation of astronomical localization, the celestial tangent plane coordinates and the CCD image coordinates of the star are included, and there is a rough error in the star data. In order to compare the effects of robust weighted mixed least squares algorithms on data processing, a set of data is simulated. Constructors $u=-5 x+8 y+6, v=8 x+5 y+2$. Select a set of data

TABLE.1 Initial data

\begin{tabular}{ccccc}
\hline No & $x$ & $y$ & $u$ & $v$ \\
\hline 1 & 1.0 & 3.0 & 25.0 & 25.0 \\
2 & 1.8 & 4.0 & 29.0 & 36.4 \\
3 & 2.0 & 1.5 & 8.0 & 25.5 \\
4 & 2.0 & 3.0 & 20.0 & 33.0 \\
5 & 2.2 & 1.7 & 8.6 & 28.1 \\
6 & 2.5 & 2.0 & 9.5 & 32.0 \\
7 & 3.0 & 1.5 & 3.0 & 33.5 \\
\hline
\end{tabular}

Add some error to the data, and add a coarse error to the fourth data.

TABLE.2 The data including error

\begin{tabular}{ccccc}
\hline No & $x$ & $y$ & $u$ & $v$ \\
\hline 1 & 1.01 & 3.02 & 24.8 & 24.8 \\
2 & 1.82 & 4.10 & 29.2 & 36.38 \\
3 & 2.11 & 1.52 & 7.80 & 25.48 \\
4 & 2.80 & 3.02 & 20.02 & 32.98 \\
5 & 2.25 & 1.71 & 8.58 & 28.23 \\
6 & 2.47 & 1.98 & 9.47 & 32.04 \\
7 & 2.97 & 1.51 & 2.92 & 32.90 \\
\hline
\end{tabular}

The least squares, the least squares, the mixed least squares and the robust weighted mixed least squares pair coefficients are used respectively.

TABLE.3 The results of different methods

\begin{tabular}{ccccc}
\hline Methods & $\hat{a}$ & $\hat{b}$ & $\hat{c}_{1}$ & $\hat{c}_{2}$ \\
\hline Least squares method & 4.2141 & 7.7683 & 5.2631 & 3.1279 \\
Total least squares method & 4.7735 & 6.9289 & 8.9130 & 3.7995 \\
Mixed least squares method & 4.4753 & 8.2497 & 4.6792 & 1.4376 \\
Robust weighted mixed least squares & 4.9107 & 7.8284 & 6.6536 & 1.3175 \\
\hline
\end{tabular}

For astronomical positioning, the conversion factor between the celestial plane coordinate system and the CCD image coordinate system will directly affect the solution center of the rotation center. So the accuracy of the coordinate conversion coefficient requirements is extremely important. To estimate the sum of the difference between the value of the parameter and the actual 
value as an important index of the evaluation method, that is

$$
\delta=\sum_{i}\left(\hat{\boldsymbol{x}}_{i g}-\boldsymbol{x}_{i z}\right)^{2}
$$

Compare the least squares, the total least squares, the mixed least squares, and the robust weighted mixed least squares algorithm can be used to derive the values in table 4 .

\begin{tabular}{cc} 
Table.4 The comparison of different methods \\
\hline Methods & $\delta$ \\
\hline Least squares method & 2.4866 \\
Total least squares method & 12.9221 \\
Mixed least squares method & 2.3985 \\
Robust weighted mixed least squares & 0.9340 \\
\hline
\end{tabular}

It can be clearly seen from the table that the value of the robust weighted mixed least squares algorithm is the smallest, indicating that the parameter value calculated using the robust weighted least squares algorithm is closest to the actual value and the highest accuracy. Followed by mixed least squares, least squares and total least squares algorithms, respectively, which represent the advantages of robust weighted mixed least squares algorithm.

\section{CONCLUSION}

In the process of astronomical positioning using the digital zenith camera, the solution of the coordinate system conversion parameter is very important for the solution of the station position.In this paper, the least squares algorithm and the total least squares algorithm are combined to form a mixed least squares algorithm, and the effect of coarse error on the solution result is eliminated by robust weighting. Through the analysis of the data, it is shown that the robust least weight algorithm is more accurate in astronomical positioning, and the calculated parameter value is closer to the actual value.

\section{REFERENCES}

[1] R.Schoebel, G.W.Hein, B.Eissfeller. Renaissance of Astrogeodetic Leveling Using CPS/CCD Zenith Camera[C]. Proceeding of the IAIN World Congress in association with the U.S.ION ANNUAL MEETING. 2000, 732-740

[2] TianLiLi,GuoJinYun,HanYanBen,et al. Digital zenith telescope prototype of China[J].Chin.Sci. Bull,2014,59(12): 1094- 1099

[3] Schaffrin B, Wieser A. Total Least-squares Adjustment of Condition Equations[J]. Studia Geophysicaet Geodaetica,2011.55(3): 529-536.

[4] GONG Xunqiang; LI Zhilin. A Robust Mixed LS-TLS Based on IGGII Scheme[J]. Geomatics and Information Science of Wuhan University ,2014,39(4):462-466.

[5] TAO Yeqing, GAO Jinxiang, YAO Yifei. A solution to plane cooedinate transformation based on robust total least sqares[J]. Journal of China University of Mining\&Technology, 2014,43(3): 534-539.

[6] Kukush A and Huffel SV. Consistency of elementwise weighted total least squares estimator in a Multrivariate Errors-In-Variables Model AX=B[J]. Springer-Verlag Metrika,2004,59:57-97.

[7] GONG Xunqiang; LI Zhilin. A Robust Weighted Total Least Sqares Method[J]. Acta Geodaetica et Cartographica Sinica, 2014, 39(4):888-894. 